\title{
Schema Modes and Personality Disorder Symptoms in Alcohol-Dependent and Cocaine-Dependent Patients
}

\author{
Michiel Booga, ${ }^{a}$ Klaartje M. van Hest ${ }^{a}$ Tamar Drescher ${ }^{c}$ Margot J. Verschuur ${ }^{d}$ \\ Ingmar H.A. Franken ${ }^{\text {b }}$ \\ a Bouman Mental Health Care, Rotterdam, The Netherlands; ' Institute of Psychology, Erasmus University Rotterdam,

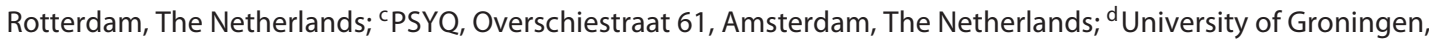 \\ Groningen, The Netherlands
}

\section{Keywords}

Substance use disorder · Cocaine - Alcohol · Schema modes $\cdot$ Schema therapy

\begin{abstract}
Substance use disorders (SUD) and personality disorders cooccur frequently. This relationship might be understood by studying schema modes (a key concept in Schema therapy), which explain the dysfunctions characterizing personality disorder patients. In the present study, we compared the schema modes and personality disorder symptoms between alcohol-dependent patients, cocaine-dependent patients and healthy controls. We found indications that specific schema modes are specific for SUD patients. However, no differences between specific subtypes of SUD patients (alcohol-vs. cocaine-dependent patients) could be found regarding schema modes. Further, it is suggested that borderline personality disorder symptoms are highly relevant for SUD patients. A first step is made in understanding the relationship between schema modes and SUD, which may contribute to the understanding of the problematic behaviour seen
\end{abstract}

\begin{tabular}{ll}
\hline KARGER & $\begin{array}{l}\text { (c) } 2018 \text { The Author(s) } \\
\text { Published by S. Karger AG, Basel }\end{array}$ \\
E-Mail karger@karger.com & $\begin{array}{l}\text { This article is licensed under the Creative Commons Attribution- } \\
\text { NonCommercial-NoDerivatives 4.0 International License (CC BY- } \\
\text { NC-ND) (http://www.karger.com/Services/OpenAccessLicense). } \\
\text { Usage and distribution for commercial purposes as well as any dis- } \\
\text { tribution of modified material requires written permission. }\end{array}$
\end{tabular}

in patients with personality disorders and SUD (and may possibly contribute to the improvement of the treatment of this group of patients).

(c) 2018 The Author(s)

Published by S. Karger AG, Basel

\section{Introduction}

Substance abuse is a very common disorder [1] worldwide; it is associated with great economic costs, and emotional, social and financial problems [2, 3]. Recovering from substance abuse is associated with frequent relapses $[4,5]$ and treatment drop-out [6]. Several reasons for this relapse and drop-out have been documented; one reason might be the profile of personality traits in substance use disorder (SUD) patients and the high comorbidity rates of personality disorders in patients with SUD [7-9]. Regarding basic dimensions of personality, an SUD profile

M.B. and K.M.H. contributed equally to the work. 
is marked by high disinhibition/impulsivity scores, and low levels of conscientiousness and agreeableness [1012]. The personality trait of disinhibition has been found to be an important predictor of SUD, both in cross-sectional as well in prospective studies [13]. Further, in a review of Verheul et al. [14], it was concluded that about $44 \%$ of people abusing alcohol and about $70 \%$ of individuals abusing cocaine have personality disorders (in both patient and non-patient samples). Antisocial and borderline personality disorder (ASPD and BPD) are the most prevalent personality disorders (with a prevalence rate of about 20\%) [15]. These comorbid personality disorders hinder treatment, for example, treatment outcomes are worse than those of SUD patients without comorbidity $[15,16]$. Furthermore, comorbid personality disorders are associated with impaired therapeutic relationships [17] and higher chances of relapse after treatment [18].

A promising and relative new treatment approach for personality disorders is Schema therapy. This is an evidence-based form of psychotherapy for various personality disorders [19-21], which has its roots in the schema theory of Young et al. [19]. According to Young et al. [19], schemas are "self-defeating emotional and cognitive patterns that begin early in our development and repeat through our life". Behavioural reactions are not included in the schema concept [22]. Schema modes are the combination of one or more activated schemas and the associated coping reaction. The concept of schema modes is developed in attempts to explain the "flipping" (the frequent and abrupt changing in mood and behaviour) seen in complex (mainly borderline personality disordered) patients [22, 23]. Young et al. [19] define a schema mode as "an enduring facet or part of the self that has not been fully integrated with other facets or parts of the self". Severe pathology comes with extreme forms of disintegration of these schema modes [22], and individuals with severe pathology have little control over the frequent flipping between different modes. Fourteen modes have been identified [24]. Targeting these schema modes in therapy (instead of the more stable schemas) is indicated in treatment of severely disturbed patients [23, 25]. Promising therapeutic results have been reported in borderline patients using the schema modes approach $[21,26]$.

The schema theory and therapy also seem to be a relevant perspective for the treatment of SUD patients. For example, it has been found that high scores on the Young Schema Questionnaire are associated with substance use problems in both clinical [27-32] and non-clinical samples [33]. Strikingly, only in one of these studies, person-

Schema Modes and Personality Disorders in Substance Abuse Patients ality disorders have been taken into account [28]. This is remarkable because the schema concept stems from theories on treatment of personality disorders. Therefore, it might well be the case that the relevance of schemas for SUD lies in the high level of co-occurrence of personality disorders and SUD.

We are not aware of studies addressing the presence of schema modes in SUD. In contrast, there is some research conducted on the applicability of Schema therapy in SUD (Ball [34]) by evaluating the effectiveness of Dual Focus Schema Therapy (DFST). DFST is an adapted form of schema therapy that is specifically designed to address SUD and personality disorders together. These studies come to contradictory findings regarding the effectiveness of DFST. Ball et al. [37] find a positive effect of DFST on therapy utilization in patients with personality disorders and SUD, and Ball [38] concludes that DFST is more effective in reducing substance use and establishing a therapeutic alliance than 12 Step Facilitation Therapy in opioid patients with personality disorders. However, in a third trial [36], no evidence was found for the effectiveness of DFST. One of the issues of the latter study, as discussed by Lee and Arntz [39], is that the researchers only focused on schemas but did not target schema modes, which would arguably had been more effective.

The present study aims to identify schema modes that are specific for alcohol-dependent and cocaine-dependent individuals. This way the relevance of schema modes for SUD can possibly be revealed further, which might inform current schema-based treatments for SUD. Because of the complex pathology of patients with SUD and personality disorders, and the preference of targeting schema modes instead of schemas in SUD therapy, schema modes are the main interest in this study. Since schema modes and SUD both have a clear behavioural component (substance use is often seen as a coping mechanism in schema theory), investigating schema modes (instead of schemas) becomes even more legitimate. As far as we know, this is the first study that examines the relationship between schema modes and SUD.

Although the relevance of the concept of schema modes in SUD patients seems evident, the theoretical underpinnings are in its infancy. A conceptual starting point can be found in a model postulated by Kersten [40]. Kersten suggests that SUD originates from the schema modes "Self-Aggrandizer" (see Table 1 for different schema modes; the names of different schema modes are chosen in such a way that they are appealing and intuitive to patients. For definitions of different schema modes, see [41]), "Bully and Attack", "Detached Protector", "De-

Eur Addict Res 2018;24:226-233 
Table 1. Schema modes
Vulnerable child
Angry child
Enraged child
Impulsive child
Undisciplined child
Happy child
Compliant surrender
Detached protector
Detached self-soother
Self-aggrandizer
Bully and attack
Punitive parent
Demanding parent
Healthy adult

tached Self Soother", "Vulnerable Child", "Angry Child", "Impulsive Child" and "Punitive Parent". Kersten suggests that "Detached Protector" and "Detached Self Soother" are characteristic for alcohol-dependent individuals and that the schema modes "Self-Aggrandizer" and "Bully and Attack" are related to cocaine abuse. Because of the explorative nature of the present study, we do not focus exclusively on these hypothesized differences between cocaine-and alcohol-dependent patients. Hence, we hypothesize that SUD patients have higher scores on the schema modes "Detached Protector", "Detached Self Soother", "Self-Aggrandizer", "Bully and Attack", "Vulnerable Child", "Angry Child", "Impulsive Child" and "Punitive Parent" as compared to controls.

To further study the relevance of the above-mentioned schema modes for cocaine and alcohol dependent patients, the relationship between the severity of substance dependence and these schema modes is investigated.

Lastly, we also investigate personality disorders, in order to characterize our sample, and to compare the 3 groups regarding personality disorders. We focus on borderline personality disorder and antisocial personality disorder (because these are the most prevalent personality disorders in SUD and because of the focus on these personality disorders in Kersten's model). Based on prior research [14], we expect a higher prevalence of personality disorders symptoms in SUD patients as compared to controls.

\section{Method}

\section{Participants}

One hundred and fifty-nine individuals participated in this study. The sample consisted of 3 groups: alcohol-dependent patients, cocaine-dependent patients and healthy controls. Partici- pants were recruited in outpatient facilities of 2 mental health care services (Bouman Mental Health Care and Ready for Change, Rotterdam, the Netherlands). Recruitment of healthy controls was conducted through convenience sampling. Characteristics of the groups are displayed in Table 2. Patients with concomitant axis I diagnoses (according to DSM-IV-TR criteria; [42]) were not included, also regarding concomitant SUD diagnoses (alcohol-dependent patients were excluded in case of a concomitant SUD; cocaine-dependent patients were excluded too if a concomitant SUD was diagnosed). Healthy controls did not suffer from any Axis I disorder whatsoever. Sufficient knowledge of Dutch language was required for inclusion.

\section{Procedure}

Patients were recruited after a standard intake procedure. Recruitment was based on diagnoses assessed during the intake procedure (by independent psychiatrists and clinical psychologists according to DSM-IV-TR criteria) [42]. Healthy controls were recruited using convenience sampling via the network of the researchers. One of the members of the research team approached potential participants and asked them to volunteer, if they met criteria for inclusion. In the 2 patient groups, collection of data took place after a period of at least 3 weeks of abstinence of all substances. Data collection took place in a single session. A member of the research team assessed demographic characteristics, executed an interview and administered two questionnaires. All participants who participated in the study provided informed consent. The study protocol has been approved by the committee on human research of Antes.

\section{Measures}

The Screening Questionnaire Personality Disorders (Vragenlijst Kenmerken Persoonlijkheid [VKP]; [43]) measures (criteria of) personality disorders according to the DSM-IV-TR. The VKP is a self-report instrument that consists of 197 items, clustered in 12 DSM-IV-TR scales. The items are phrases like "I trust most people" or "I have good friends"; respondents are asked to state whether they agree with a particular statement. Two dependent variables are generated: a dimensional score (the sum score of the criteria met per personality disorder) and a diagnosis (a score above threshold concerning a specific personality disorder). In this study, the dimensional score is regarded as main outcome because of the loss of information in generating a categorical diagnostic variable. The psychometric qualities of the VKP are reasonable [43]. The reliability of the VKP scales is acceptable (Cronbach's vary between 0.59 and 0.78 ; average 0.66 ).

The Schema Mode Inventory (SMI; [24]) is a self-report instrument that is used for schema mode assessment. The SMI is composed of 14 scales, representing 14 schema modes. The SMI has acceptable internal consistency (Cronbach's from 0.79 to 0.96 ), adequate test-retest reliability, and moderate construct validity.

The severity of substance abuse was measured by means of the Addiction Severity Index (ASI), section III (alcohol and drugs use) [44]. The ASI is a structured interview that assesses, among others, the types of drugs used, number of years of use, and age of onset of use. Psychometric qualities of the ASI are acceptable [44]. The ASI was administered in all 3 groups (it was applied in the control group as well, to check once more no SUD-patients were included in this group). Two ASI variables were used: age of first frequent use and proportion of years of frequent use (years of frequent use divided by age). 
Table 2. Characteristics of subjects

\begin{tabular}{|c|c|c|c|}
\hline & Alcohol-dependent group & Cocaine-dependent group & Healthy controls \\
\hline Gender & 39 males, 17 females & 41 males, 6 females & 34 males, 22 females \\
\hline Level of education ${ }^{\mathrm{a}}, \%$ & $\begin{array}{l}1=33.9 \\
2=26.8 \\
3=39.3\end{array}$ & $\begin{array}{l}1=25.5 \\
2=44.7 \\
3=29.8\end{array}$ & $\begin{array}{l}1=8.9 \\
2=25.0 \\
3=66.1\end{array}$ \\
\hline Any personality disorder & $42.9 \%$ & $63.8 \%$ & $17.9 \%$ \\
\hline $\begin{array}{l}\text { Two personality disorders } \\
\text { with highest mean score } \\
\text { (dimensional) }^{\mathrm{b}}\end{array}$ & $\begin{array}{l}\text { Antisocial }(\text { Mean }=2.61 \\
\mathrm{SD}=2.81) \\
\text { Borderline }(\text { Mean }=2.5 \\
\mathrm{SD}=2.30)\end{array}$ & $\begin{array}{l}\text { Borderline }(\text { Mean }=4.04 \\
\mathrm{SD}=2.11) \\
\text { Antisocial }(\text { Mean }=3.57 \\
\mathrm{SD}=4.12)\end{array}$ & $\begin{array}{l}\text { Obsessive- compulsive } \\
(\text { Mean }=1.52, \mathrm{SD}=1.49) \\
\text { Paranoid }(\text { Mean }=1.04, \\
\mathrm{SD}=1.45)\end{array}$ \\
\hline
\end{tabular}

${ }^{a}$ Level of education: 1 = low (elementary to middle school), 2 = intermediate (high school), 3 = high (college or university).

${ }^{b}$ Number of PD criteria met.

${ }^{\mathrm{c}}$ Individuals with a specific personality disorder.

\section{Data Analysis}

In order to investigate whether the cocaine-dependent, the alcohol-dependent and the healthy control groups differed in respect to level of education, a chi-square test for independence was conducted. To investigate possible differences between the 3 groups regarding gender, Fisher's exact test was used (because of unequal distribution between cells). Age differences between the 3 groups were evaluated using a one-way analysis of variance.

The differences in prevalence between the 3 groups concerning BPD and ASPD (the 2 most prevalent personality disorders) were regarded by means of an analysis of covariance (ANCOVA), with demographic variables as covariates.

To examine whether the alcohol group, the cocaine group and the healthy control group differed regarding schema modes, ANCOVA's were executed, with demographic variables as covariates. Finally, the relationships between specific schema modes and severity of addiction were investigated by means of correlational analyses.

\section{Results}

\section{Demographic Characteristics}

A chi-square test for independence made clear that there was a significant association between group (cocaine, alcohol and controls) and level of education; the effect size was medium, $\chi^{2}(4)=19.87, p=0.001$, Cramer's $\mathrm{V}=0.25$. The control group had the highest level of education.

Schema Modes and Personality Disorders in Substance Abuse Patients
A Fisher's exact test was conducted regarding gender. The groups differed significantly regarding gender $(p=$ $0.008)$ : the cocaine group had the lowest $(12.7 \%)$ proportion of women, the control group had the highest (39.3\%) proportion of women.

A one-way ANOVA pointed out that the alcohol group, cocaine group and control group differed significantly in age: $F(2,156)=6.08, p=0.003$. Post hoc comparisons (Tukey HSD) made clear that the mean age for alcohol patients (47.39 years) was significantly higher than the mean age of cocaine patients (39.79 years). The control group did not differ significantly in age (43.43 years) from either alcohol- or cocaine-dependent patients.

\section{Personality Disorders}

Significant differences between the 3 groups were found concerning BPD and ASPD dimensional scores: ANCOVA's (with gender, age and level of education as covariates. ANCOVA's were followed by pairwise comparisons based on estimated means) showed that the cocaine-dependent patients had a higher BPD dimensional score than alcohol-dependent patients and controls; alcohol-dependent patients had a higher BPD score than normal controls as well $(F[2,153]=24.29$, $p=0.001$, partial eta squared $=0.24$, a large effect size according to Cohen [45]. Cocaine-dependent and alco- 
Table 3. ANCOVA's schema modes $(n=159)$

\begin{tabular}{lccll}
\hline & $F(\mathrm{df})$ & $p$ value & Partial eta squared & Pairwise comparison \\
\hline "Detached self-soother" & $10.68^{*}(2,153)$ & $\leq 0.001$ & 0.12 (medium effect size) & Cocaine/alcohol $>$ control \\
"Detached protector" & $3.92(2,153)$ & 0.02 & 0.05 (small) & Cocaine $>$ control \\
"Self-aggrandizer" & $0.56(2,153)$ & 0.57 & 0.01 & - \\
"Bully and attack" & $1.71(2,153)$ & 0.18 & 0.02 & - \\
"Vulnerable child" & $9.99^{*}(2,153)$ & $\leq 0.001$ & $0.12($ medium) & Cocaine/alcohol $>$ control \\
"Angry child" & $7.27^{*}(2,153)$ & $\leq 0.001$ & 0.09 (medium) & Cocaine $>$ control \\
"Impulsive child" & $8.51^{*}(2,153)$ & $\leq 0.001$ & 0.10 (medium) & Cocaine/alcohol $>$ control \\
"Punitive parent" & $11.05^{*}(2,153)$ & $\leq 0.001$ & 0.13 (medium) & Cocaine/alcohol $>$ control \\
\hline
\end{tabular}

* Significant after Bonferroni adjustment.

hol-dependent patients did not differ in ASPD dimensional scores; both patient groups, however, had significant higher ASPD dimensional scores than healthy controls $(F[2,153]=7.02, p=0.001$, eta squared $=0.08$ (moderate effect size).

As an additional robustness check, non-parametric correlations were computed between the level of education and BPD and ASPD dimensional scores in the patient group (cocaine-dependent and alcohol-dependent patients together) and in the control group. In the patient group, a significant correlation was found between the level of education and BPD dimensional score, $r_{s}=-0.37$, $p=0.005$, with a high level of education associated with lower BPD dimensional scores. No further significant correlations were found.

\section{Schema Modes}

ANCOVAs were conducted to compare the differences between the alcohol-dependent patients, cocaine-dependent patients and controls regarding the schema modes "Detached Self-Soother", "Detached Protector", "Self-Aggrandizer", "Bully and Attack", "Vulnerable child", "Angry Child', "Impulsive Child", and "Punitive Parent". In each ANCOVA age, gender and level of education were used as covariates. Because of the relatively great number of comparisons, Bonferroni adjustment was employed. Alpha levels of.00625 per test (0.05/8) were used (Table 3).

Again, additional non-parametric correlations were computed between the level of education and the aforementioned schema modes in the patient group (cocainedependent and alcohol-dependent patients together) and the control group. In the control group, a significant correlation was found between the level of education and the schema mode "Self-Aggrandizer", $r_{s}=0.37, p=0.005$,
Table 4. Correlations between schema modes and substance use variables in alcohol-dependent patients $(n=56)$

\begin{tabular}{lll}
\hline & $\begin{array}{l}\text { Age of first } \\
\text { frequent use of } \\
\text { alcohol }>5 \text { units }\end{array}$ & $\begin{array}{l}\text { Proportion } \\
\text { years of } \\
\text { frequent use } \\
\text { of alcohol }>5 \\
\text { units }\end{array}$ \\
\hline "Detached protector" & -0.10 & 0.25 \\
"Detached self-soother" & -0.13 & 0.19 \\
"Self-aggrandizer" & -0.29 & 0.11 \\
"Bully and attack" & -0.17 & 0.01 \\
"Vulnerable child" & -0.25 & $0.43^{\mathrm{b}}$ \\
"Angry child" & -0.36 & $0.44^{\mathrm{b}}$ \\
"Impulsive child" & -0.09 & 0.10 \\
"Punitive parent" & -0.14 & 0.33 \\
\hline
\end{tabular}

${ }^{a}$ Years of frequent use is divided by age.

${ }^{\mathrm{b}}$ Significant after Bonferroni adjustment.

with high level of education associated with high "SelfAggrandizer" scores. No further significant correlations were found in this respect.

Finally, correlations were computed between substance use variables and the above-mentioned schema modes ("Detached Self Soother", "Detached Protector", "Self-Aggrandizer", "Bully and Attack", "Vulnerable Child", "Impulsive Child", "Angry Child" and "Punitive Parent"). Because of the relatively great number of comparisons, Bonferroni correction was applied. Alpha levels of.00625 per test $(0.05 / 8)$ were used. Significant positive associations were observed between schema modes "Vulnerable child" and "Angry child" and "proportion of frequent use of alcohol $>5$ units" in alcohol-dependent patients (Tables 4, 5). 
Table 5. Correlations between schema modes and substance use variables in cocaine-dependent patients $(n=47)$

\begin{tabular}{lll}
\hline & $\begin{array}{l}\text { Age of first } \\
\text { frequent use } \\
\text { of cocaine }\end{array}$ & $\begin{array}{l}\text { Proportion } \\
\text { years of } \\
\text { frequent use } \\
\text { of cocaine }\end{array}$ \\
\hline "Detached protector" & 0.16 & -0.09 \\
"Detached self-soother" & 0.02 & 0.05 \\
"Self-aggrandizer" & -0.12 & 0.12 \\
"Bully and attack" & 0.00 & 0.03 \\
"Vulnerable child" & -0.01 & -0.10 \\
"Angry child" & 0.03 & -0.01 \\
"Impulsive child" & 0.15 & -0.07 \\
"Punitive parent" & 0.11 & -0.18 \\
\hline
\end{tabular}

a Years of frequent use is divided by age.

\section{Discussion}

In the present study, the differences between alcoholdependent patients, cocaine-dependent patients and healthy individuals were examined regarding specific schema modes.

The 3 groups differed in scores on "Detached SelfSoother", "Vulnerable Child", "Angry Child", "Impulsive Child" and "Punitive Parent" (medium effect sizes). Except for the mode "Angry Child", both cocaine patients and alcohol patients obtained higher scores than controls on these schema modes. For the "Angry Child" schema mode, only cocaine patients had higher scores than controls. No differences between cocaine patients and alcohol patients were found in any of the investigated schema modes.

The findings of the present study partly support the hypotheses based on Kersten's model [40], which suggests 8 schema modes to be characteristic for SUD patients. Differences were found between SUD patients and healthy controls on the schema modes "Detached SelfSoother", "Vulnerable Child", "Angry Child", "Impulsive Child", and "Punitive Parent". However, contrary to expectations based on Kersten's model, no differences were found between SUD patients and controls on "Detached Protector", "Self-Aggrandizer" and "Bully and Attack". Further, Kersten [40] suggests the existence of schema modes that would be typical for cocaine-dependent patients and alcohol-dependent patients. However, the present data do not support this hypothesis, but rather suggest that these schema modes are relevant for SUDs in general.

Schema Modes and Personality Disorders in Substance Abuse Patients
Having said this, the present data provided some putative clues for some more substance-specific subtypes. Interestingly, positive relationships between the schema modes "Vulnerable Child" and "Angry Child" and severity of substance abuse were found in the alcohol group (but not in the cocaine group), which suggests that these modes might be especially relevant for alcohol-dependent patients.

Concerning the general prevalence of personality disorders in alcohol and cocaine patients, the present study shows a largely comparable prevalence to the Verheul et al. [14] study. More specific, the prevalence of ASPD and BPD diagnoses - the 2 most prevalent personality disorders for SUD - in the current study was very similar to prevalence figures found in earlier research [14], suggesting that our patient sample was representative in terms of personality disorders. An exception could be the relative high prevalence of paranoid personality disorder in our cocaine patients. In our study, the 3 groups differed from each other concerning the prevalence of ASPD and BPD. In addition, alcohol- and cocaine-dependent patients had higher ASPD dimensional scores than normal controls; cocaine patients had higher scores on BPD than alcohol patients (and normal controls), who, in their turn, had higher BPD scores than controls. Remarkably, especially the difference in BPD criteria met was considerable (large effect size; Cohen [45]).

The present study has some limitations and strengths that should be mentioned. First, patients with concomitant axis I diagnoses were to be excluded. These axis I diagnoses were established during the intake procedure. At that moment, patients sobriety was probably incipient, and a clear view on some concomitant axis I diagnoses might have been obscured by the pharmacological effects of substance use. However, concomitant axis I diagnoses seem to recover with recovering of SUD [46]. It therefore seems unlikely that a great number of patients with concomitant axis I disorders were accidentally included in the present study. A second limitation lies in the absence of formal diagnostic investigation of healthy controls. Healthy controls were asked whether they were affected by any psychiatric disorder (and were excluded on affirmation), and their substance abuse was examined through the administration of the ASI. But the existence of DSMIV-TR diagnoses was not formally confirmed. Third, personality disorders were established by a self-report measure, whereas semi-structured interviews are the preferred method for assessing personality disorders [47]. Fourth, we acknowledge that background variables such as educational level were not perfectly matched between the groups and additional analyses showed that educa- 
tional level was in some occasions related to schema modes and personality disorder symptoms. Although we controlled for these variables in the analyses, future studies should include larger and better matched samples.

To conclude, SUD patients have higher scores on specific schema modes than healthy controls, and these schema modes might be helpful in discriminating SUD patients from healthy individuals and understanding the psychological dynamics of substance abuse. For now, schema modes seem to be of limited value in differentiating between cocaine- and alcohol-dependent patients because no differences were found on schema modes between cocaine-dependent and alcohol-dependent patients. In clinical practice, it seems important to assess every individual patient and develop a treatment plan that matches that particular patient (irrespective of preferred substance of abuse). The effect size of the difference on the BPD scores was large: BPD symptoms seem to be highly characteristic for SUD patients. Future research is needed to find out what the relative value is of the assessment of BPD symptoms and schema modes. Do these 2 concepts have a unique value in understanding
SUD patients, and especially in treating their problems? For now, in light of the research done on SUD and personality, there is far more and stronger evidence for the relevance of basic personality traits (conscientiousness, agreeableness, disinhibition, e.g., $[11,12])$ and personality disorders [7] in characterizing SUD patients. Further research should make clear whether addressing schema modes in therapy for SUD patients might be a valuable addition to current therapy practice. This use of schema modes might be clinically relevant because the schema mode concept and schema therapy bring clear tools for therapists in working with complex pathology.

\section{Disclosure Statement}

All authors declare that they have no conflicts of interest to disclose.

\section{Funding Source}

There was no external funding involved in this study.

\section{References}

1 Kessler RC, Berglund P, Demler O, Jin R, Merikangas KR, Walters EE: Lifetime prevalence and age-of-onset distributions of DSMIV disorders in the national comorbidity survey replication. Arch Gen Psychiatry 2005;62: 593-602.

2 Kessler RC, Foster CL, Saunders WB, Stang PE: Social consequences of psychiatric disorders, I: educational attainment. Am J Psychiatry 1995; 152:1026-1032.

3 Andlin-Sobocki P, Rehm J: Cost of addiction in Europe. Eur J Neurol 2005;12:28-33.

4 Gossop M, Stewart D, Browne N, Marsden J: Factors associated with abstinence, lapse or relapse to heroin use after residential treatment: protective effect of coping responses. Addiction 2002;97:1259-1267.

5 Sinha R: The role of stress in addiction relapse. Curr Psychiatry Rep 2007;9:388395.

6 Brorson HH, Ajo Arnevik E, Rand-Hendriksen K, Duckert F: Drop-out from addiction treatment: a systematic review of risk factors. Clin Psychol Rev 2013;33:1010-1024.

7 Zimmerman M, Rothschild L, Chelminski I: The prevalence of DSM-IV personality disorders in psychiatric outpatients. Am J Psychiatry 2005;162:1911-1918.

8 Walcott G, Martin J, Hickling F: The prevalence of personality disorder in a psychiatric and substance abuse population in Jamaica. West Indian Med J 2013;62:458-462.
9 Trull TJ, Sher KJ, Minks-Brown C, Durbin J, Burr R: Borderline personality disorder and substance use disorders: a review and integration. Clin Psychol Rev 2000;20:235-253.

10 Walton KE, Roberts BW: On the relationship between substance use and personality traits: abstainers are not maladjusted. J Res Pers 2004;38:515-535.

11 Kotov R, Gamez W, Schmidt F, Watson D: Linking "big" personality traits to anxiety, depressive, and substance use disorders: a metaanalysis. Psyc Bull 2010;136:768-821.

12 Conway KP, Kane RJ, Ball SA, Poling JC, Rounsaville BJ: Personality, substance of choice, and polysubstance involvement among substance dependent patients. Drug Alcohol Depend 2003;71:65-75.

13 Sher KJ, Bartholow BD, Wood MD: Personality and substance use disorders: a prospective study. J Consult Clin Psychol 2000;68:818-829.

14 Verheul R, van den Brink W, Hartgers C: Prevalence of personality disorders among alcoholics and drug addicts: an overview. Eur Addict Res 1995;1:166-177.

15 Verheul R, Bosch W, Ball S: Verslaving en persoonlijkheidspathologie; in EurelingsBontekoe EHM, Verheul R, Snellen WM (eds): Handboek Persoonlijkheidspathologie. Houten, Bohn Stafleu van Loghum, 2009, pp 417-432.

16 Compton WM, Cottler LB, Jacobs JL, BenAbdallah A, Spitznagel EL: The role of psychi- atric disorders in predicting drug dependence treatment outcomes. Am J Psychiatry 2003; 160:890-895.

17 Verheul R, van den Brink W, Hartgers C: Personality disorders predict relapse in alcoholic patients. Addict Behav 1998;23:869-882.

18 Thomas VH, Melchert TP, Banken JA: Substance dependence and personality disorders: comorbidity and treatment outcome in an inpatient treatment population. J Stud Alcohol 1999;60:271-277.

19 Young JE, Klosko JS, Weishaar ME: Schema Therapy: A Practitioner's Guide. Guilford Press, 2003

20 Bamelis LL, Evers SM, Spinhoven P, Arntz A: Results of a multicenter randomized controlled trial of the clinical effectiveness of schema therapy for personality disorders. Am J Psychiatry 2014;171:305-322.

21 Giesen-Bloo J, van Dyck R, Spinhoven P, van Tilburg W, Dirksen C, van Asselt T, Kremers I, Nadort M, Arntz A: Outpatient psychotherapy for borderline personality disorder: randomized trial of schema-focused therapy vs transference-focused psychotherapy. Arch Gen Psychiatry 2006;63:649-658.

22 Sempértegui GA, Karreman A, Arntz A, Bekker MH: Schema therapy for borderline personality disorder: a comprehensive review of its empirical foundations, effectiveness and implementation possibilities. Clin Psychol Rev 2013;33:426-447. 
23 Lobbestael J, van Vreeswijk M, Arntz A: Netherlands Journal of Psychology, 2007, number 3 Shedding light on schema modes: a clarification of the mode concept and its current research status. Neth J Psychol 2007;63: 69-78.

24 Lobbestael J, van Vreeswijk M, Spinhoven P, Schouten E, Arntz A: Reliability and validity of the short schema mode inventory (SMI). Behav Cogn Psychother 2010;38:437-458.

25 Arntz A, Jacob G: Schematherapie. Een Praktische Handleiding. Amsterdam, Uitgeverij Nieuwezijds, 2012.

26 Nadort M, Arntz A, Smit JH, Giesen-Bloo J, Eikelenboom M, Spinhoven P, van Asselt T, Wensing M, van Dyck R: Implementation of outpatient schema therapy for borderline personality disorder with versus without crisis support by the therapist outside office hours: a randomized trial. Behav Res Ther 2009;47: 961-973.

27 Ball SA, Cecero JJ: Addicted patients with personality disorders: traits, schemas, and presenting problems. J Pers Disord 2001;15:7283.

28 Shorey RC, Anderson S, Stuart GL: The relation between antisocial and borderline personality symptoms and early maladaptive schemas in a treatment seeking sample of male substance users. Clin Psychol Psychother 2014;21:341-351.

29 Brotchie J, Meyer C, Copello A, Kidney R, Waller G: Cognitive representations in alcohol and opiate abuse: the role of core beliefs. Br J Clin Psychol 2004;43:337-342.

30 Roper L, Dickson JM, Tinwell C, Booth PG, McGuire J: Maladaptive cognitive schemas in alcohol dependence: changes associated with a brief residential abstinence program. Cogn Ther Res 2010;34:207-215.

31 Shorey RC, Stuart GL, Anderson S: Differences in early maladaptive schemas between a sample of young adult female substance abusers and a non-clinical comparison group. Clin Psychol Psychother 2014;21:21-28.

32 Shorey RC, Stuart GL, Anderson S: Early maladaptive schemas among young adult male substance abusers: a comparison with a non-clinical group. J Subst Abuse Treat 2013;44:522-527.

33 Bakhshi Bojed F, Nikmanesh Z: Role of early maladaptive schemas on addiction potential in youth. Int J High Risk Behav Addict 2013; 2:72-76.

34 Ball SA: Manualized treatment for substance abusers with personality disorders: dual focus schema therapy. Addict Behav 1998;23:883891.

35 Ball SA: Personality traits, problems, and disorders: clinical applications to substance use disorders. J Res Pers 2005;39:84-102.

36 Ball SA, Maccarelli LM, LaPaglia DM, Ostrowski MJ: Randomized trial of dual-focused vs. single-focused individual therapy for personality disorders and substance dependence. J Nerv Ment Dis 2011;199:319-328.

37 Ball SA, Cobb-Richardson P, Connolly AJ, Bujosa CT, O’Neall TW: Substance abuse and personality disorders in homeless drop-in center clients: symptom severity and psychotherapy retention in a randomized clinical trial. Compr Psychiatry 2005;46:371-379.

38 Ball SA: Comparing individual therapies for personality disordered opioid dependent patients. J Pers Disord 2007;21:305-321.

39 Lee CW, Arntz A: A commentary on Ball et al's (2011) study on dual focused versus single focused therapy for personality disorders and substance dependence: what can we really conclude? The Journal of Nervous and Mental Disease 2013.

40 Kersten T: Schema therapy in personality disorders and addiction; in Vreeswijk van $\mathrm{M}$, Broersen J, Nadort M (eds): The Wiley-Blackwell Handbook of Schematherapy. Chicester, John Wiley \& Sons, 2012, pp 415-424.

41 Vreeswijk van M, Broersen J, Nadort M (eds): The Wiley-Blackwell handbook of schematherapy. Chichester, John Wiley \& Sons, 2012.

42 APA: DSM-IV-TR: Diagnostic and Statistical Manual of Mental Disorders, Text Revision. Washington, American Psychiatric Association, 2000, p 75.

43 Duijsens IJ, Eurelings-Bontekoe EHM, Diekstra RW: The VKP, a self-report instrument for DSM-III-R and ICD-10 personality disorders: construction and psychometric properties. Pers Individ Dif 1996;20:171182.

44 Hendriks VM, Kaplan CD, van Limbeek J, Geerlings P: The addiction severity index: reliability and validity in a dutch addict population. J Subst Abuse Treat 1989;6:133-141.

45 Cohen J: Statistical power analysis for the behavioral sciences o Hilsdale. NJ, Lawrence Earlbaum Associates 1988, p 2.

46 Verheul R, Kranzler HR, Poling J, Tennen H, Ball S, Rounsaville BJ: Axis I and Axis II disorders in alcoholics and drug addicts: fact or artifact? J Stud Alcohol 2000;61:101-110.

47 McDermut W, Zimmerman M, Oldham J, Skodol A, Bender D: Assessment instruments and standardized evaluation. The American Psychiatric Publishing textbook of personality disorders 2005:89-101. 